Produto \& Produção, vol. 5, n. 3, p. 01-16, out. 2001

\title{
Projeto e análise de processos de serviços: uma avaliação de técnicas de representação
}

\author{
Luciano Costa Santos, M.Eng. \\ Programa de Pós-Graduação em Engenharia de Produção - UFSC \\ e-mail: lcostas@eps.ufsc.br
}

Gregorio Varvakis, Ph.D.

$C I N \& P P G E P-U F S C$

e-mail: grego@eps.ufsc.br

\begin{abstract}
O projeto e a análise dos processos de serviços podem ser desempenhados com a utilização de técnicas que visam dar suporte para tal. No entanto, não se pode afirmar que todas as técnicas utilizadas sejam adequadas para este fim. Este artigo procura apresentar e avaliar algumas técnicas utilizadas para projeto e análise de processos de serviços, com ênfase na representação de processos. Esta avaliação é feita com base em requisitos que são propostos previamente. Estes requisitos partem do pressuposto que a técnica deverá dar suporte à melhoria da qualidade nos processos de serviços, considerando suas características específicas. Depois da definição dos requisitos, são apresentadas nove técnicas utilizadas em projeto e/ou análise de processos de serviços. Ao final, é feita uma comparação entre as técnicas analisadas, utilizando como base os requisitos propostos.
\end{abstract}

Palavras-chave: processos de serviços; técnicas de representação de processos; melhoria da qualidade.

The design and analysis of service processes can be performed using techniques to support it. However, few techniques have a specific approach to the characteristics of service processes. This paper aims to present and evaluate some techniques utilized in the design and analysis of service processes, focusing the process representation. This evaluation is based on requirements that are previously defined and are needed to support the quality improvement in service processes. After the requirements definition, nine techniques for design and analysis of service processes are showed. Finally, a comparison is made between the selected techniques relating them to the defined requirements.

Keywords: service processes; process representation techniques; quality improvement.

\section{Introdução}

Com o crescimento do setor de serviços na economia, muitos profissionais ligados à Engenharia de Produção passaram a voltar suas atenções para a gestão de operações de serviços, sendo que grande parte das publicações na área tem como foco principal a melhoria da qualidade. Entretanto, mesmo com o crescente interesse na área, observa-se que a qualidade na maioria dos serviços ainda apresenta níveis inferiores à qualidade dos produtos manufaturados. Um dos fatores que levam a esta situação é a falta de uma abordagem que seja específica para as operações de serviços. Muitas vezes, procura-se aplicar nos serviços, técnicas utilizadas em operações de manufatura, mas sem considerar que os processos de manufa- tura apresentam características diferentes dos processos de serviços.

A qualidade dos serviços depende diretamente da gestão dos processos que os produzem. É importante considerar que a sobreposição entre produto e processo nas operações de serviços é maior que nas operações de manufatura. Fitzsimmons \& Fitzsimmons (1998) chegam a declarar que, em serviços, o processo é o produto. O fato é que o cliente de serviços faz parte do processo de transformação, o que torna difícil a separação entre projeto do produto e projeto do processo. Porém, não há dúvida que uma boa descrição dos processos de serviços é imprescindível tanto para a melhoria de processos existentes quanto para projetar novos serviços. 
O projeto e a análise dos processos de serviços podem ser desempenhados com a utilização de técnicas que visam dar suporte para tal. Contudo, não se pode afirmar que todas as técnicas utilizadas em projeto e análise de processos de serviços sejam adequadas para este fim. Muitas das técnicas utilizadas não consideram as especificidades das operações de serviços e falham no suporte à melhoria da qualidade nos processos.

Este artigo procura apresentar e avaliar algumas técnicas utilizadas para projeto e análise de processos de serviços, com ênfase na representação de processos. É comum encontrar na literatura publicações que fazem um levantamento das técnicas de modelagem ou mapeamento de processos de negócios (Bal, 1998; Kettinger et al., 1997; Miers, 1996), mas não especificamente representação de processos de serviços. Dentre as técnicas selecionadas neste artigo, algumas correspondem a técnicas de modelagem de processos para quaisquer tipos de operações (fluxograma tradicional, IDEF0), mas com aplicações em serviços. Outras correspondem a técnicas que foram especialmente desenvolvidas ou adaptadas para processos de serviços (service blueprint, mapa do serviço, estrutura de processamento de clientes, walk-through-audit, análise da transação de serviço, IDEF3 adaptado, linguagem de representação para projeto de processos de serviços).

A avaliação das técnicas analisadas é feita com base em requisitos que são propostos previamente. Estes requisitos partem do pressuposto de que a técnica deverá dar suporte à melhoria da qualidade nos processos de serviços, considerando suas características específicas. Depois de propor requisitos, são apresentadas nove técnicas utilizadas em projeto e/ou análise de processos de serviços. Ao final, é feita uma comparação entre as técnicas analisadas, utilizando como base os requisitos propostos.

\section{Requisitos da técnica}

As técnicas utilizadas para descrever processos de serviços, sejam para fins de projeto ou análise, terão diferentes requisitos, de acordo com os objetivos a que se propõem. Muitas técnicas poderão dar maior ênfase no projeto, enquanto outras poderão se concentrar mais na análise do processo. Entretanto, existem alguns requisitos comuns que são desejáveis, mesmo para diferentes finalidades.

Harrington et al. (1997), que trabalharam na documentação dos processos empresariais, de serviços e de manufatura, definiram seis critérios para selecionar uma técnica de documentação de processos:
- Objetivos e fatores. Os objetivos da documentação irão determinar os fatores a serem documentados, bem como o nível de detalhe requerido. Conseqüentemente, isto irá influenciar diretamente na escolha da técnica de documentação.

- Facilidade de uso. A técnica deverá ser fácil de usar, tanto pela pessoa que irá elaborar o documento, como pela pessoa que irá utilizar o documento.

- Documentação existente. É necessário considerar que, quando já existe alguma documentação de processos em uma organização, as pessoas da organização já estão familiarizadas com a técnica, o que irá pesar na escolha.

- Manutenibilidade. A facilidade de manutenção, ou seja, a capacidade da técnica em permitir mudanças na documentação com simplicidade, é um importante critério de escolha.

- Intensidade de trabalho. Está diretamente relacionada com esforço necessário para desenvolver e manter um processo. A técnica não pode demandar tanto trabalho para descrever o processo a ponto de não se obter o retorno necessário.

- Subjetividade. Uma técnica pode ser considerada melhor do que outra devido a critérios subjetivos de escolha, como o fato das pessoas estarem mais familiarizadas ou terem um conhecimento maior em relação a uma determinada técnica.

Além dos critérios para selecionar uma técnica de documentação, Harrington et al. (1997) apresentam sugestões gerais para o uso de técnicas de diagramação. Dentre elas:

- A documentação deverá estar sintonizada com as necessidades do usuário.

- A documentação deverá ter a maior facilidade de uso possível.

- O uso de símbolos deve ser limitado, particularmente para um leitor menos experiente.

- O texto nos diagramas deverá ser claro e conciso.

- Os diagramas devem ter uma organização visual clara.

Já no contexto do projeto de serviços, Gummesson citado por Congram \& Epelman (1995), identificou quatro critérios que uma metodologia de descrição de processos de serviços deve apresentar para que seja eficaz. Congram \& Epelman (1995) acrescentaram mais quatro critérios aos identificados por Gummesson, propondo um total de oito critérios: 
1. A apresentação gráfica do modelo deve ser compreensível para os empregados.

2. A metodologia deve ter uma linguagem clara e consistente.

3. O modelo deve ser baseado em atividades e orientado para a ação.

4. A gerência deve apoiar o uso da metodologia.

5. Os empregados devem participar no processo de desenvolvimento do modelo.

6. O modelo deve auxiliar os empregados a realizar suas tarefas mais eficazmente.

7. A metodologia deve guiar a gerência de serviços nos esforços relacionados com o controle do processo.

8. A metodologia deve favorecer a colaboração entre as funções de operações, marketing e recursos humanos.

Ma (1999) também propôs requisitos para uma linguagem de representação de projeto de processos de serviços. $\mathrm{O}$ autor dividiu estes requisitos em duas categorias: requisitos funcionais e critérios técnicos. Os requisitos identificados por Ma (1999) são os seguintes:

\section{Requisitos funcionais:}

- Facilitar a documentação do projeto de processos de serviços.

- Dar suporte à verificação do projeto.

- Facilitar o entendimento e comunicação do projeto de processos de serviços entre as partes envolvidas.

\section{Critérios técnicos:}

- Ser específica para processos de serviços.

- Manter a formalidade apropriada.

- O processo deve ser possível de ser representado em qualquer nível de detalhe.

- Manter interpretação não-ambígua.

- Ser simples e fácil de usar.

Pode-se observar que os requisitos apresentados por diferentes autores muitas vezes se sobrepõem, o que favorece a identificação de características comuns entre as diferentes técnicas de projeto e análise de processos de serviços.

\subsection{Requisitos propostos}

Baseando-se nos critérios apresentados pelos autores citados (Harrington et al., 1997; Congram \& Epelman, 1995; Ma, 1999) e nas características dos serviços, propõe-se alguns requisitos desejáveis para uma técnica de projeto e análise de processos de serviços.

1. Adequação tanto para o projeto quanto para a análise de processos de serviços. Uma técnica de representação de processos de serviços deve servir de base tanto para o projeto, quanto para análise de processos. Quando o processo do serviço é projetado com uma determinada técnica, é interessante que se utilize a mesma técnica na análise do processo, o que permite alterar facilmente o projeto do processo. Assim, a técnica seria útil não só na fase de projeto, mas na gestão do serviço em todas as fases, apoiando o ciclo "planejamento/ implementação/controle/ajuste".

2. Descrição da experiência de serviço do ponto de vista do cliente. A técnica deve ser capaz de mapear o fluxo de clientes no processo, descrevendo as atividades que o cliente participa. Isto auxiliaria os gerentes de serviço a entender como o cliente vê o processo de prestação de serviço. É interessante lembrar, que para descrever com precisão a experiência que o cliente tem durante a prestação do serviço, a técnica deve permitir a representação da variabilidade dos processos de serviços, em um nível de detalhamento adequado.

3. Representação gráfica baseada em diagramas. Mesmo que a linguagem natural seja direta e fácil de entender, ela permite uma certa ambigüidade e dificulta a modelagem de situações do mundo real (Ma, 1999). Como afirma Miers (1996, p. 176), "um diagrama bem-feito, como uma imagem, vale por mil palavras". Na verdade, um diagrama facilita a compreensão do processo, tanto para fins de projeto como de análise. Portanto, torna-se necessária uma técnica baseada na combinação de elementos gráficos e texto, apresentados de uma forma organizada e sistemática.

4. Facilidade de uso. A técnica deve ter facilidade de elaboração e facilidade de compreensão. A simplicidade da técnica e a clareza da linguagem irão influenciar fortemente neste requisito. A facilidade de uso deve permitir que mesmo os gerentes nãoespecialistas possam utilizar e compreender a técnica. Isto também poderia estimular a participação dos empregados e a comunicação do processo 
na empresa, uma vez que todos na organização teriam facilidade de compreender o processo.

5. Suporte para a avaliação de desempenho do processo. Para apoiar a melhoria do processo, é desejável que a técnica permita a avaliação do desempenho em cada atividade. A identificação de problemas no processo de prestação de serviço normalmente passa por uma constatação de que o desempenho em determinados pontos de interação está abaixo do desejado. Assim, é fundamental para a fase de análise do processo de serviço que a técnica dê suporte para a avaliação de desempenho.

\section{Técnicas de projeto e análise de processos de serviços}

\subsection{Fluxograma tradicional}

Existem várias derivações do fluxograma tradicional, conforme a finalidade que ele é utilizado. Para a descrição detalhada do processo, ele pode ser chamado de diagrama detalhado de processo (Harrington et al., 1997) ou simplesmente de fluxograma (Miers, 1996). O nome fluxograma tradicional refere-se ao fluxograma no seu formato original, uma vez que as derivações do fluxograma tradicional também podem ser chamadas de fluxograma, pois de certa forma descrevem o "fluxo" do processo.

Em geral, os fluxogramas descrevem a seqüência de atividades de um processo empresarial. Para isso, os fluxogramas utilizam uma simbologia padronizada que adota, entre outros símbolos, retângulos para representar atividades, losangos para representar pontos de decisão e setas para indicar o sentido de fluxo. Estes símbolos vêm acompanhados de textos que descrevem as atividades e orientam o fluxo do processo.

Harrington (1993) considera que a função básica do fluxograma é documentar um processo para permitir a identificação das áreas a serem aperfeiçoadas. De fato, o fluxograma é de grande utilidade para a representação de processos. Porém, se tratando de processos de serviços, ele possui muitas limitações.

Apesar do fluxograma ser simples e fácil de usar, seus recursos gráficos não permitem uma descrição simples de processos com grande divergência. De acordo com Shostack (1987), a divergência de um processo está relacionada com a latitude de execução ou a variabilidade na seqüência de atividades. O fluxograma também não tem recursos para descrever processos paralelos, ou seja, processos que podem ocorrer simultaneamente. Outra desvantagem é que os fluxogramas se tornam grandes demais ao representar processos com grande complexidade. A complexidade de um processo se refere ao número de atividades envolvidas (Shostack, 1987). Este problema se deve ao fato do fluxograma tradicional representar o processo sem fazer distinção no nível de detalhamento, ao invés de agregar as atividades em níveis de detalhe diferentes, decompondo as atividades de níveis de detalhe mais altos em atividades de níveis de detalhe mais baixos.

Entretanto, a maior desvantagem do fluxograma tradicional para a representação de processos de serviços é que ele considera o processo do ponto de vista da empresa e não do cliente. Assim, o processo é descrito segundo as atividades desempenhadas pela empresa, e mesmo que estas atividades envolvam o consumidor, a percepção do cliente não é considerada.

\subsection{Service blueprint}

Apesar de ser derivado dos fluxogramas usados na gestão de processos industriais, o service blueprint se diferencia destes, pois considera o aspecto da interação com o consumidor. Segundo Brown et al. (1994), uma diferença primária entre o service blueprint e o fluxograma tradicional é que o blueprint incorpora o cliente e as ações do cliente no mesmo fluxograma do resto da operação. Desta forma, o processo é visto sob a perspectiva do cliente e não da empresa.

De acordo com Fitzsimmons \& Fitzsimmons (1998), o blueprint é um mapa de todas as transações que constituem o processo de entrega do serviço. Este mapa identifica tanto as atividades de linha de frente como as atividades de retaguarda, separadas pela linha de visibilidade (Figura 1).

A idéia lançada pelo service blueprint foi tão bem assimilada, que esta técnica ainda é bastante aceita e utilizada com poucas modificações, sendo citada por grande parte das publicações recentes na área de gestão de serviços (Téboul, 1999; Fitzsimmons \& Fitzsimmons, 1998; Ramaswamy, 1996). Nas diversas publicações, encontra-se o diagrama com diferentes nomes e diferentes aplicações, porém todos incorporam a idéia original do service blueprint de mapear o processo do ponto de vista do cliente.

Quando o conceito do service blueprint surgiu, já era sugerida a utilização da técnica na identificação dos pontos de falha no processo (Shostack, 1984). Rotondaro \& Oliveira (1999) utilizaram o service blueprint para analisar cada atividade da linha de frente em relação aos determinantes da qualidade em serviços envolvidos e em relação a um indicador do potencial de falha que foi desenvolvido. Já Chase \& 


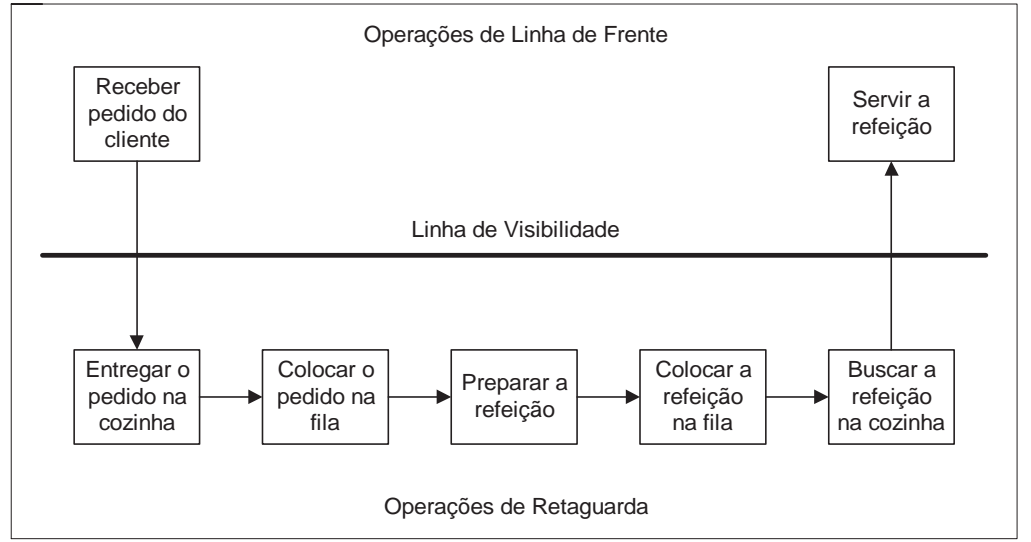

Figura 1 - Exemplo de um service blueprint para o processo de entrega de refeições em um restaurante Fonte: Adaptado de Ramaswamy (1996)

Stewart (1994), utilizaram o service blueprint para identificar falhas potenciais em cada atividade, com o objetivo de sinalizar a incorporação de dispositivos à prova de falhas (poka-yokes) no processo.

Schmenner (1995) propõe a aplicação do service blueprint na identificação dos gargalos do processo, planejamento da capacidade e tempos de execução, análise dos custos envolvidos, entre outros. Lovelock (1995) ainda acrescenta a esta lista o replanejamento do projeto de trabalho. Segundo Téboul (1999), o fluxograma do serviço (conforme denomina o autor) também é útil para focalizar a empresa nas operações, melhorando a produtividade e reduzindo custos, perdas e tempos de resposta. Gianesi \& Corrêa (1994) analisam o service blueprint com a intenção de identificar os processos-chave, para em seguida estabelecer a relação destes processos com as áreas de decisão da empresa. Assim, pode-se definir quais são as áreas de decisão prioritárias para o atendimento dos objetivos operacionais estratégicos da organização. Em um outro trabalho, Shostack (1987) ainda sugeriu a aplicação do service blueprint na análise e definição do posicionamento estratégico de uma empresa de serviços.

Normalmente, o service blueprint apresenta a mesma simbologia e os mesmos recursos gráficos do fluxograma tradicional, sendo que algumas vezes ele é apresentado sem uma simbologia definida. Por isso, ainda que tenha muitas vantagens, o service blueprint apresenta as mesmas limitações do fluxograma tradicional, uma vez que ele foi derivado do fluxograma. Tseng et al. (1999) afirmam que pelo fato de ter a mesma estrutura do fluxograma tradicional, o service blueprint não está focalizado na descrição da completa experiência do cliente e, além disso, não é capaz de detalhar a informação sobre a experiência que o cliente tem durante a prestação do serviço. Congram \& Epelman (1995) também criticam o service blueprint, alegando que ele revela os problemas inerentes da abordagem de fluxogramas. Estes autores questionam a ambigüidade e a inconsistência no significado dos símbolos e ainda criticam os níveis de detalhe inconsistentes que ocorrem no diagrama.

Para Johnston (1999), mesmo que o service blueprint considere as interações com o cliente, muitas vezes o processo é documentado sob a perspectiva da empresa (por exemplo, na Figura 1). O autor ainda afirma que a técnica é orientada para a tarefa e não para o cliente, pois está focalizada nas ações e eventos observáveis (tarefas).

\subsection{Mapa do serviço}

Uma derivação interessante do service blueprint é o mapa do serviço (Figura 2), proposto por KingmanBrundage et al. (1995). A grande diferença entre o mapa do serviço e o service blueprint é que ele envolve a gestão do serviço como um todo, e não somente o processo de entrega do serviço.

O mapa do serviço tenta integrar as várias dimensões envolvidas na gestão do serviço, desde a gerência da empresa até o cliente. Para isso, ele divide os processos de serviços não somente em duas partes (linha de frente e retaguarda), mas em cinco diferentes zonas. As cinco zonas são:

- Zona do cliente, onde se encontram as atividades e decisões desempenhadas pelo cliente.

- Zona da linha de frente, onde estão situadas as atividades realizadas pelos empregados que têm contato direto com o cliente.

- Zona da retaguarda, que corresponde às atividades realizadas pelos empregados que apoiam diretamente à linha de frente.

- Zona de suporte, que envolve as atividades que suportam às três zonas anteriores. 


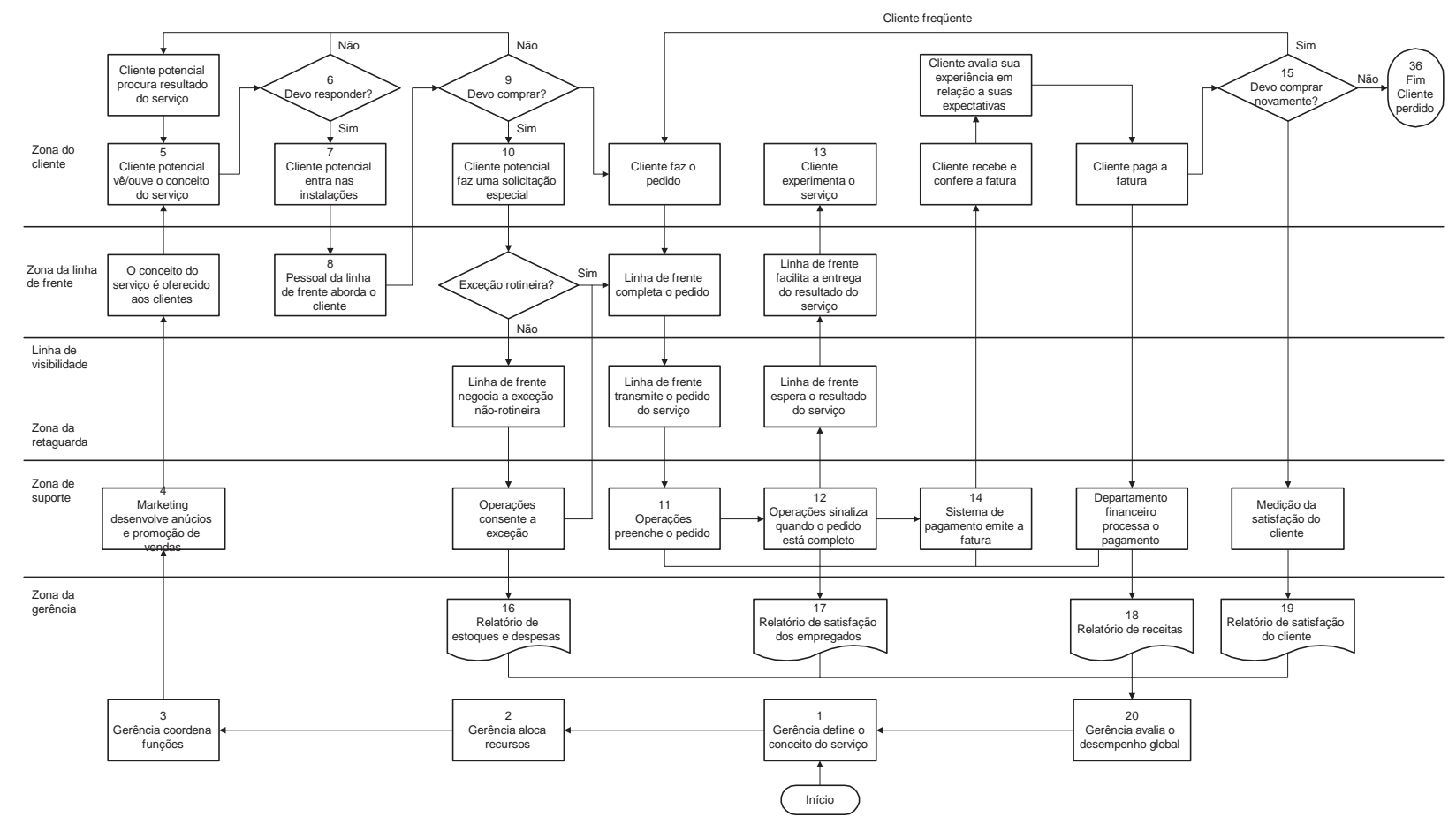

Figura 2 - Mapa do serviço Fonte: Kingman-Brundage et al. (1995, p. 28)

- Zona da gerência, que engloba as atividades que a gerência deve realizar para apoiar o encontro de serviço.

A linha de visibilidade divide as duas primeiras zonas das três últimas. No mapa do serviço o cliente ocupa o topo do diagrama, a gerência ocupa a base, enquanto as operações de serviços se localizam no meio. De acordo com Kingman-Brundage et al. (1995), o mapa do serviço mostra como o pessoal de serviços manipula os componentes do serviço para fechar a lacuna entre a gerência e o cliente.

Entre outras aplicações, o mapa do serviço foi utilizado por Kingman-Brundage et al. (1995) para descrever a "lógica" do serviço, por meio de um modelo genérico que compreende a maioria das atividades de qualquer processo de serviços (como é o caso da Figura 2). Neste modelo, os autores discutiram vinte atividades-chave, analisando suas implicações para a gestão de serviços.

Mesmo com uma maior abrangência que o service blueprint no que se refere à gestão do serviço como um todo, o mapa do serviço não apresenta grandes diferenças com o service blueprint no que se refere ao projeto e análise de processos. Por isso, ele possui as mesmas desvantagens que o service blueprint, para fins de projeto e análise de processos. Johnston (1999) considera que o mapa do serviço é um refinamento do service blueprint, mas mesmo assim, afirma que ele é orientado para a tarefa e não para o cliente.

\subsection{Estrutura de processamento de clien- tes}

Outra técnica de diagramação utilizada para projetar processos de serviços é a estrutura de processamento de clientes, apresentada por Slack et al. (1997). Ao invés de mapear as atividades específicas de cada tipo de serviço, como fazem o service blueprint e o mapa do serviço, a estrutura de processamento de clientes propõe um modelo genérico de atividades-chave que são comuns à maioria dos processos de serviços. Assim, o processo do serviço pode ser projetado por meio do planejamento de cada atividade genérica em cada caso particular.

É importante observar que esta técnica visa especificamente o fluxo de clientes, identificando apenas as atividades que envolvem o cliente. Desta forma, o diagrama só mostra as atividades de linha de frente. $\mathrm{Na}$ estrutura de processamento de clientes são identificadas sete atividades-chave (ver Figura 3), que ocorrem na seguinte seqüência:

- Seleção: é momento em que o cliente decide escolher a operação de serviço.

- Ponto de entrada: é o primeiro contato com a operação escolhida.

- Tempo de resposta: é o tempo que o cliente espera até que o sistema responda.

- Ponto de impacto: é o momento em que o cliente começa a ser atendido. 
- Prestação de serviço: é a parte em que o serviço principal é prestado.

- Ponto de partida: é o ponto em que o cliente deixa o processo do serviço.

- Acompanhamento: são todas as atividades de acompanhamento do cliente após a conclusão do serviço.

As atividades-chave facilitam o trabalho de projeto e análise dos processos de serviços, dado que, para cada caso particular, os gerentes de serviços terão que planejar (projeto) ou reavaliar (análise) cada atividade genérica. No entanto, a tentativa de estabelecer atividades genéricas em uma sequiência rígida, e não atividades específicas para cada caso, mesmo que facilite o trabalho de projeto e análise, também o limita.

De fato, o modelo gerado pela estrutura de processamento de clientes torna-se muito distante da realidade quando se consideram processos de serviços específicos. Os próprios autores afirmam: "Quando examinadas em detalhe, as operações de serviços, em sua maior parte, compreendem diversas seqüências de processamento de clientes, que podem ser em série ou em paralelo" (Slack et al., 1997, p. 160). Somente esta afirmação já demonstra que a rigidez da estrutura de processamento de clientes torna este diagrama incapaz de descrever com precisão a experiência do cliente.

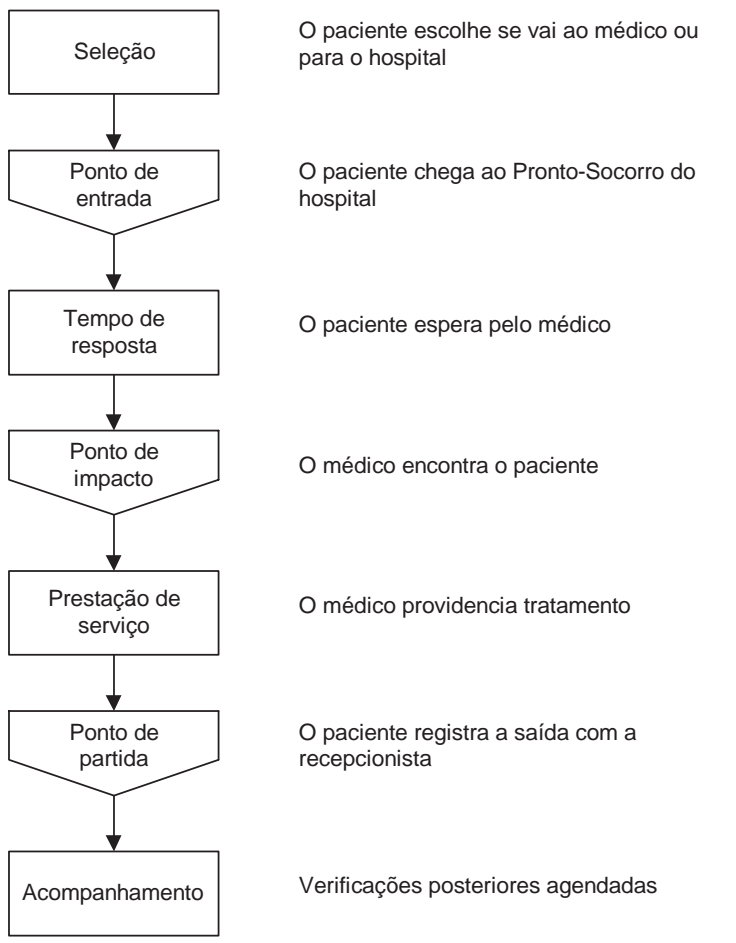

Figura 3 - Exemplo da estrutura de processamento de clientes para o Pronto-Socorro de um hospital

Fonte: Slack et al. (1997, p. 162)

\subsection{IDEFØ}

À medida que o conceito do service blueprint foi evoluindo, técnicas mais sofisticadas de mapeamento de processos foram sendo aplicadas em operações de serviços. Um bom exemplo é a aplicação do $I D E F O$, uma técnica que foi inicialmente desenvolvida para processos industriais. De acordo com Mayer et al. (1999), o IDEF0 é uma técnica derivada de uma linguagem gráfica conhecida como Structured Analysis and Design Technique (SADT), sendo que freqüentemente se encontram na literatura os dois nomes se referindo à mesma técnica (Congram \& Epelman, 1995). O IDEF0 foi desenvolvido pela Força Aérea dos Estados Unidos com o objetivo de descrever, especificar e modelar sistemas de manufatura (Plaia \& Carrie, 1995). No entanto, Congram \& Epelman (1995) demonstraram ser bastante viável a aplicação desta técnica na descrição de processos de serviços.

A simbologia utilizada no IDEF0 é constituída por retângulos, que representam as atividades ou os processos, e setas, que representam os recursos e informações que são necessários para executar as atividades. Esses recursos podem ser entradas, saídas, mecanismos e controles, posicionados em torno de cada atividade como mostra a Figura 4. As entradas representam os recursos utilizados pela atividade para produzir as saídas. As saídas são os resultados produzidos por cada atividade. Os mecanismos são os elementos usados para executar a atividade. Os controles representam informações que restringem ou regulam a atividade, ou seja, que afetam a forma como a atividade é executada. Plaia \& Carrie (1995) relacionam as entradas e saídas com a pergunta "O que é feito?", os mecanismos com a pergunta "Como é feito?", e os controles com a pergunta "Por que é feito?".

Segundo Mayer et al. (1999), o IDEF0 não somente representa as atividades individualmente, mas revela as relações entre as atividades de um processo. As saídas de uma atividade podem se tornar as entradas, os controles ou até mesmo os mecanismos da atividade seguinte, dispostos em um diagrama que começa no topo à esquerda e termina na base à direita (Figura 5). Outra importante característica do IDEF0 é o princípio de decomposição hierárquica das atividades. Cada atividade de um diagrama do IDEF0 pode ser decomposta em atividades menores, sendo que a decomposição pode ser feita até o nível de detalhe que for requerido para cada aplicação. 


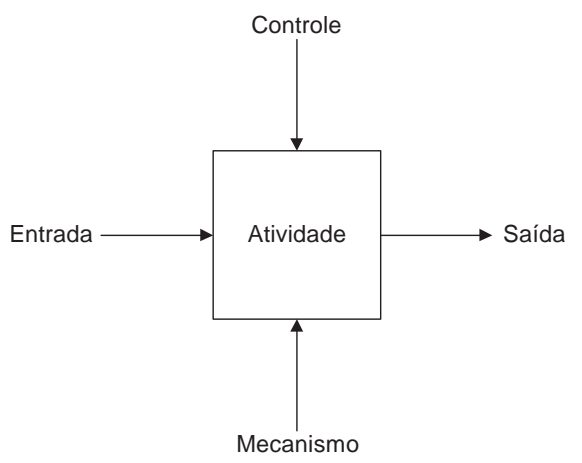

Figura 4 - Elementos do IDEF0

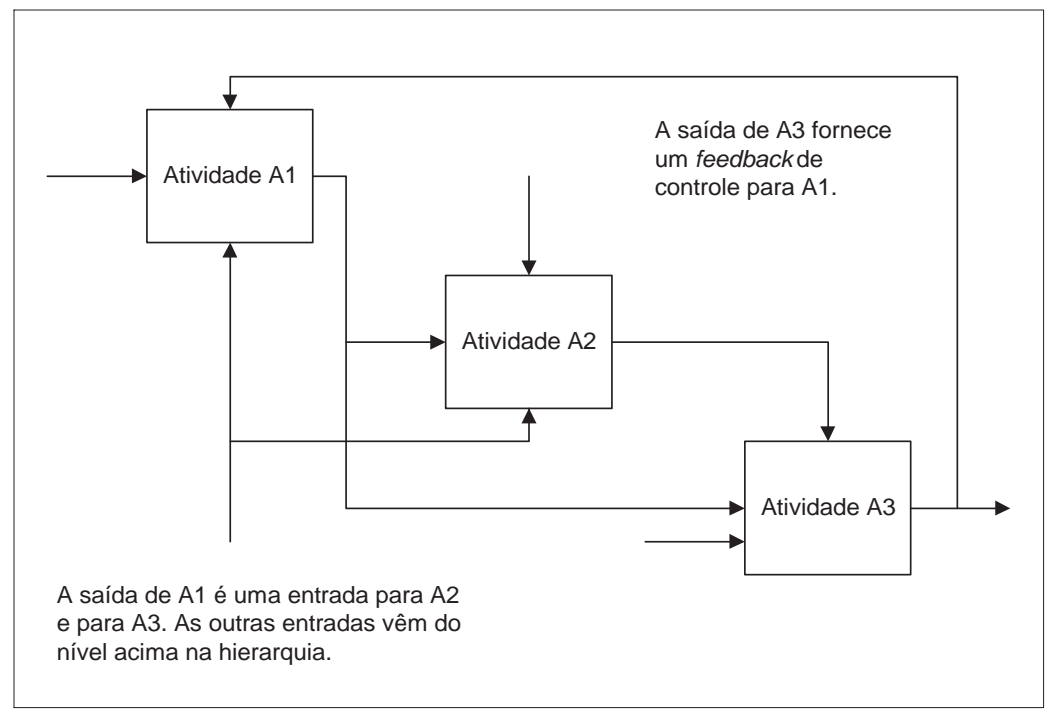

Figura 5. O diagrama IDEF0

Fonte: Bal (1998, p. 344)

Uma qualidade do IDEF0 é a precisa identificação dos recursos envolvidos no processo, quer sejam recursos humanos, materiais ou informações. O IDEF0 descreve "o que" uma organização faz, o que leva a empresa a questionar se as atividades que ela desempenha são realmente relevantes (Mayer et al., 1999). No entanto, não há uma lógica específica, nem uma noção de tempo associado ao processo (Plaia \& Carrie, 1995). Isto prejudica a descrição da experiência do cliente, já que o encontro de serviço é composto por uma seqüência de atividades de interação com o cliente. Além disso, as pessoas têm uma tendência de interpretar diagramas como uma seqüência de eventos, devido à "cultura" herdada dos fluxogramas. Este fato dificulta a compreensão do IDEF0, pois sua ênfase não está na sequiência de atividades, mas no conteúdo das atividades e nos recursos envolvidos no processo.

\subsection{Walk-through-audit}

A análise do projeto de serviços pode também incluir a análise do projeto do pacote de serviços, e não somente a análise do processo. Isto é bastante coerente, pois na verdade, a experiência do cliente durante o processo inclui todos os elementos do pacote (bens facilitadores, instalações e serviço prestado). Este é o caso da walk-through-audit (WTA), uma espécie de auditoria do serviço baseada em uma série de questões dirigidas aos clientes e gerentes de serviços, relativas ao processo e ao pacote de serviços (Fitzsimmons \& Fitzsimmons, 1998; Koljonen \& Reid, 2000). Uma importante característica da WTA é que ela analisa o processo do ponto de vista do consumidor. Segundo Fitzsimmons \& Fitzsimmons (1998), a walk-through-audit é uma ferramenta de gestão para uma avaliação sistemática da visão do cliente do serviço prestado.

A walk-through-audit utiliza questões estruturadas, onde os clientes avaliam cada etapa do processo por meio de uma escala de cinco pontos. À medida que se detalha o processo, pode-se elaborar inúmeras questões relacionadas a cada momento da verdade. A Figura 6 mostra exemplos de algumas questões que podem ser feitas na aplicação da WTA em um restau- 
rante self-service. $\mathrm{O}$ exemplo sugere uma questão para cada atividade de interação com o cliente no restaurante.

A grande diferença entre a WTA e a pesquisa de satisfação do cliente é que a WTA analisa a experiência do cliente ao longo de cada estágio do processo, e não apenas identifica a satisfação do cliente em relação ao serviço como um todo (Koljonen \& Reid, 2000). O questionário é respondido pelos clientes durante ou imediatamente após o serviço. Por isso, a WTA pode ser considerada uma técnica de análise de processos de serviços e é normalmente aplicada pelo pessoal de operações, sendo que as pesquisas de satisfação do cliente são aplicadas pelo pessoal de marketing (Koljonen \& Reid, 2000).

A WTA pode ser utilizada em conjunto com o fluxograma do processo ou qualquer técnica que representa o processo graficamente. Koljonen \& Reid (2000) apresentam um modelo para a aplicação da WTA em cinco etapas:

1. Elabore o fluxograma do processo de criação e entrega do serviço na perspectiva do cliente.

2. Planeje, teste e aplique o questionário para uma amostra de clientes, para o pessoal da gerência, e/ ou para clientes de organizações concorrentes (benchmarking).

3. Sintetize e analise os resultados dando ênfase nas pontuações baixas em relação à concorrência e nas diferenças de pontuação entre a gerência e os clientes.
4. Determine as deficiências e implemente melhorias.

5. Para a melhoria contínua, repita os passos de 1 a 4 .

Fitzsimmons \& Fitzsimmons (1998) apresentam um exemplo de aplicação da WTA em restaurantes por meio de um questionário de 42 perguntas distribuídas entre nove categorias de variáveis, dentre elas: itens de manutenção, serviço pessoal, espera, ambiente e apresentação da comida. Nesse caso, Fitzsimmons \& Fitzsimmons (1998) aplicaram o questionário somente aos proprietários ou gerentes dos restaurantes, o que reduz significativamente a validade da técnica para a análise do processo segundo a visão do cliente, embora também permita analisar o processo.

Já Koljonen \& Reid (2000) aplicaram a WTA em um escritório de advocacia, conforme o modelo de cinco etapas apresentado anteriormente. Depois da elaboração do fluxograma do processo, os autores agruparam as atividades em quatro estágios: pré-chegada, chegada e consulta. Além de elaborarem cinco questões para os estágios de pré-chegada e chegada, e dez para o estágio de consulta, os autores acrescentaram mais cinco questões para a categoria de avaliação agregada de desempenho. Em seguida, compararam as pontuações médias dos clientes com as pontuações médias: da gerência, dos clientes da concorrência e da gerência da concorrência.

Além dos benefícios de avaliar a percepção do cliente ao longo do processo e incluir na análise todos os elementos do pacote de serviços, a WTA possui a vantagem de analisar a lacuna entre as percepções do cliente e as percepções da gerência, bem como

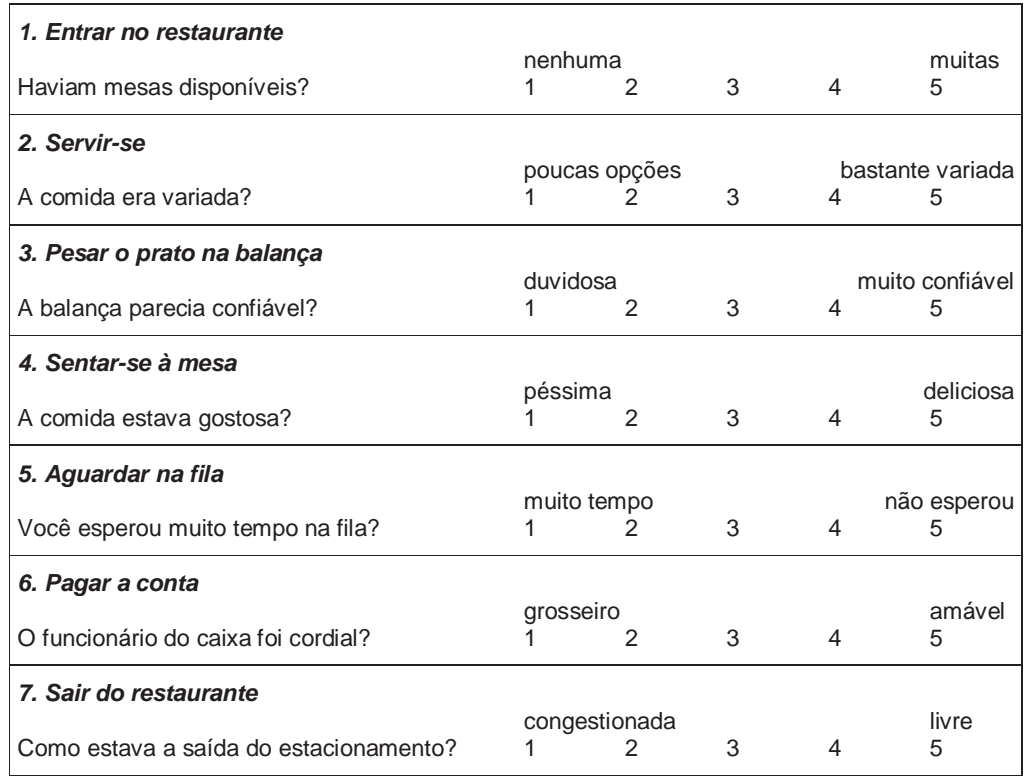

Figura 6. Exemplo de questões de uma WTA para um restaurante self-service 
analisar a lacuna entre a empresa e a concorrência.

Mesmo com todas as vantagens, Johnston (1999) afirma que a principal fraqueza da WTA é que a análise por meio de questões sistematicamente estruturadas pode comprometer a flexibilidade requerida para avaliar as percepções do cliente ao longo do processo. Outro fator, é que a WTA é uma técnica somente de análise e não de projeto. Mesmo que esta técnica possa ser utilizada em conjunto com técnicas de representação gráfica, ela por si só não apresenta uma representação gráfica do processo, o que constitui mais uma limitação.

\subsection{Análise da transação de serviço}

Como mencionado anteriormente, Johnston (1999) critica as técnicas tradicionais de mapeamento de processos de serviços, como o service blueprint e o mapa do serviço de Kingman-Brundage et al. (1995). $\mathrm{O}$ autor alega que estas técnicas estão orientadas para a tarefa e não para o cliente, descrevendo o processo de serviço da perspectiva da empresa e não do cliente. Além disso, o autor critica a rigidez do questionário da WTA. Em resposta a tudo isso, Johnston (1999) desenvolveu uma técnica denominada de análise da transação de serviço (STA - Service Transaction Analysis).

Assim como a walk-through-audit, a análise da transação de serviço avalia o processo do ponto de vista do cliente, combinando quatro elementos críticos: o conceito do serviço, o processo do serviço, a avaliação da qualidade em cada transação, e a interpretação do serviço pelo cliente (que é composta de "mensagens" emitidas em cada transação). Para sua operacionalização, a técnica utiliza um formulário denominado "folha de análise da transação de serviço”. Conforme Johnston (1999), esta técnica compreende cinco estágios:

1. O conceito do serviço é especificado.

2. Compradores fantasmas, conselheiros independentes ou clientes-consultores caminham ao longo do processo atual para analisar como o cliente poderia avaliar cada transação (qualquer interação, seja com o ambiente inanimado ou através do contato pessoal). Cada transação é descrita com poucas palavras, sendo determinado seu score (pontuação): cliente encantado (+), cliente satisfeito $(0)$, cliente insatisfeito (-).

3. As mensagens sutis emitidas em cada transação, isto é, são anotadas as interpretações que levaram o "suposto" cliente a chegar a essa avaliação.

4. Os pontos (+), (0) e (-) são interligados ao longo da folha de análise da transação de serviço, de modo a formar um gráfico de linha. Em seguida, a avaliação global é anotada.

5. Com a folha de análise da transação de serviço preenchida, os gerentes de serviço podem começar a entender como os clientes poderiam interpretar o processo, para depois discutir as melhorias a serem feitas.

Para exemplificar, Johnston (1999) apresenta o caso de um escritório de advocacia, mostrado na Figura 7. É interessante observar neste caso, que a avaliação global do processo contradiz o conceito do serviço especificado para o escritório. Além da avaliação global, as avaliações em cada transação sinalizam as melhorias que podem ser feitas no processo.

Assim como a WTA, a análise da transação de serviço avalia o pacote de serviços além de avaliar o processo. Além disso, ela induz o questionamento sobre quais as "mensagens" que a empresa tem emitido para seus clientes. Na análise da transação de serviço, tanto a avaliação como a descrição do processo são feitas pelo cliente. Mesmo que a WTA avalie o processo segundo a percepção do cliente, este processo é previamente mapeado pela empresa e não pelo cliente. Na análise da transação de serviço o processo pode ser percebido de diversas formas para diferentes clientes. Ao invés das atividades serem previamente estabelecidas, esta técnica descreve e avalia as transações que podem ser ou não percebidas em diferentes ocasiões. Desta maneira, o processo é descrito como ele realmente ocorre e como ele realmente é percebido pelo cliente.

Entretanto, mesmo com toda a simplicidade e orientação para o cliente que esta técnica possui, pode-se constatar algumas limitações. Embora bastante oportuno, o fato de utilizar pessoas que se passam por clientes (e não os próprios clientes) pode gerar algumas distorções nos resultados. Além disso, esta técnica somente pode ser utilizada na análise do processo e não no projeto. E também, apesar do gráfico gerado pelo score em cada transação, o processo não é representado graficamente, pois todas transações são anotadas por escrito.

\subsection{IDEF3 adaptado}

O mapeamento do processo do ponto de vista do cliente também pode ser feito por uma adaptação do IDEF3, proposta por Tseng et al. (1999). O IDEF3 é mais um integrante da família de técnicas IDEF, desenvolvida pela Força Aérea dos Estados Unidos. Tseng et al. (1999) fizeram algumas simplificações e adaptações para o caso específico das operações de serviços, uma vez que o IDEF3, assim como o IDEF0, foi concebido inicialmente para operações industriais. 


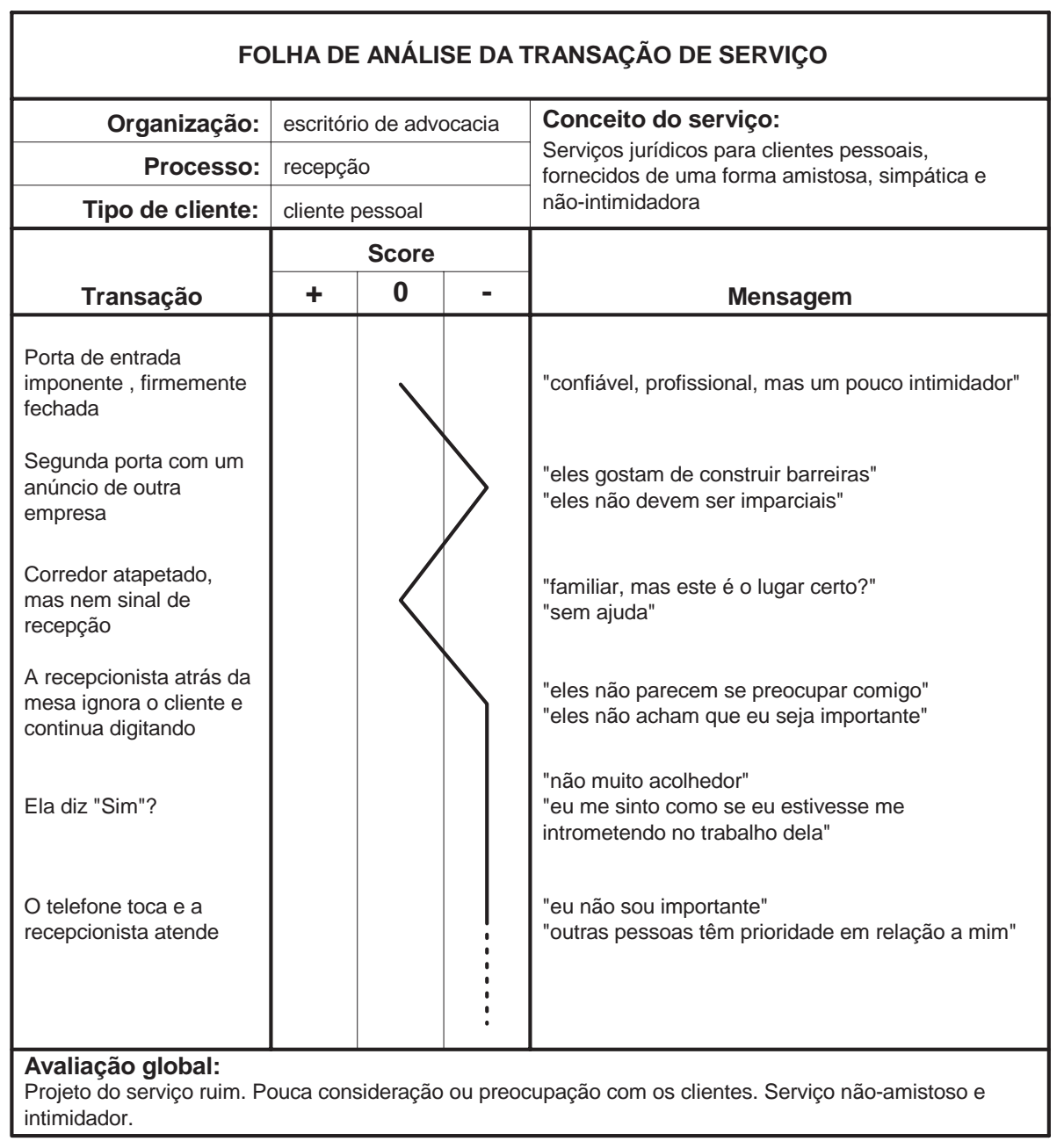

Figura 7 - Exemplo de uma análise da transação de serviço para o processo de recepção em um escritório de advocacia Fonte: Johnston (1999, p. 106)

De acordo com Plaia \& Carrie (1995), existem dois tipos de diagrama no IDEF3: o diagrama de fluxo de processo e a rede de transição de estado de objeto. Tseng et al. (1999) consideraram somente o diagrama de fluxo de processo e então fizeram algumas modificações. Ao adaptar a técnica, os autores procuraram identificar as atividades em que o cliente participa do processo, dando o nome de "unidades de atuação do cliente" (modificando o nome "unidades de comportamento" do IDEF3 original). Cada unidade de atuação do cliente (UAC) é representada por um retângulo preenchido com o nome e número da unidade. $\mathrm{O}$ diagrama do IDEF3 adaptado é então elaborado a partir das unidades de atuação do cliente, que correspondem às atividades em que o cliente participa. Assim, o diagrama mostra somente as atividades de linha de frente.

As UAC's obedecem ao mesmo princípio de decomposição hierárquica do IDEF0, podendo ser decompostas de acordo com o nível de detalhamento requerido. A descrição da experiência do cliente começa com um diagrama simples, que abrange todas as atividades do processo no mais alto nível de abstração. A Figura 8 apresenta um exemplo de aplicação da técnica em um supermercado, onde é mostrada a sequiência de atividades no mais alto nível da hierarquia.

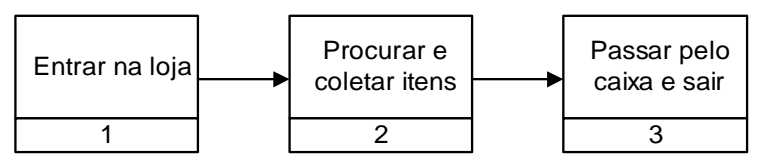

Figura 8 - Diagrama do IDEF3 adaptado Fonte: Tseng et al. (1999, p. 57)

As unidades de atuação do cliente são interconectadas por meio das setas (que indicam fluxo) e das junções. As junções são usadas para expressar sincronia ou assincronia entre as atividades e para expressar a convergência ou divergência do fluxo do processo (Plaia \& Carrie, 1995). Tseng et al. (1999) somente consideraram as junções assíncronas, ou seja, onde as atividades paralelas não precisam começar ou terminar ao mesmo tempo. Assim, os autores reduziram os dez tipos de junções do IDEF3 original para seis tipos. As junções podem ser do tipo (\&), quando 
todas atividades relacionadas com a junção acontecem, do tipo $(\mathrm{O})$, quando uma ou mais atividades relacionadas com a junção acontecem, ou do tipo $(\mathrm{X})$, quando apenas uma atividade relacionada com a junção acontece. Os três tipos de junção podem ser divergentes, quando as atividades são realizadas a partir da junção, ou convergentes, quando as atividades terminam na junção.

Outros elementos gráficos que são utilizados no diagrama do IDEF3 são os referents. Tseng et al. (1999) sugerem dois tipos de referents: "Go-to" e "Elab". O Go-to é utilizado para indicar a próxima ocorrência no processo, sem que ela esteja ligada por uma seta. Freqüentemente, o Go-to sinaliza a possibilidade do processo voltar a um ponto anterior (looping). Já o Elab, é anexado a uma junção para fornecer informações adicionais sobre o fluxo do processo.

Com a compreensão do significado das junções e dos referents, a representação gráfica do IDEF3 adaptado passa a ser de fácil utilização. À medida que se necessita detalhar mais uma UAC, pode-se decompor a atividade até onde for preciso. A Figura 9 mostra como a UAC "procurar e coletar itens" da Figura 8 foi decomposta.

Outro componente importante da técnica é o "documento de elaboração" (Figura 10). Para cada unidade de atuação do cliente existe um documento de elaboração correspondente, que fornece informações adicionais para cada unidade. No IDEF3 original, o documento de elaboração traz as seguintes informações: nome, número e rótulo da unidade de comportamento, objetos, fatos, restrições e descrição. Tseng et al. (1999) adaptaram o documento de elaboração para o caso das operações de serviços, que passou a conter as seguintes informações:

- Nome, número e rótulo da UAC: o rótulo é o nome (abreviado ou não) que está escrito no diagrama e o número vai depender do nível hierárquico da atividade.

- Objeto de contato com o cliente: são todas entidades identificáveis que entram em contato com o cliente.

- Atributos do objeto de contato com o cliente: características de um objeto que denotam propriedades que o objeto possui.

- Operações do objeto de contato com o cliente: são as operações realizadas pelo cliente ou para o cliente em cada UAC.

- Relações entre o objeto e o cliente: são as relações de troca entre o objeto e o cliente.
Ao contrário do IDEF0, o IDEF3 apresenta uma lógica específica e uma noção de tempo associado ao processo, representando a seqüência na qual ocorrem as atividades (Plaia \& Carrie, 1995). O IDEF0 se preocupa com "o que" uma organização faz, enquanto o IDEF3 foca sua atenção em "como" as coisas acontecem, sendo que em muitas situações, uma técnica complementa a outra (Mayer et al., 1999). Obviamente, as modificações feitas por Tseng et al. (1999) não alteraram estas características. A representação precisa da seqüência de atividades permite mapear a experiência que o cliente tem durante a prestação do serviço. Além disso, as informações adicionais do documento de elaboração permitem analisar tanto o processo de serviços como o pacote de serviços.

Uma limitação do IDEF3 adaptado é que ele não mostra as atividades da retaguarda, como fazem o service blueprint e o mapa do processo. Outro fator limitante, é que a técnica deixa a desejar no que diz respeito à avaliação de desempenho do processo. Mesmo que o documento de elaboração apresente informações adicionais que permitem a análise do processo, ele não tem uma relação direta com a avaliação do desempenho em cada atividade.

\subsection{Linguagem de representação para projeto de processos de serviços}

A técnica apresentada por Ma (1999) propõe uma linguagem para especificar, visualizar, construir e documentar o projeto de processos de serviços. A linguagem de representação para projeto de processos de serviços foi desenvolvida com base nos requisitos propostos pelo autor (mencionados anteriormente), nas linguagens existentes de modelagem de processos e na metodologia de modelagem orientada a objeto. Além de desenvolver uma linguagem gráfica, o autor desenvolveu uma linguagem formal correspondente à linguagem gráfica, para aplicações computacionais.

A base teórica para a linguagem desenvolvida por Ma (1999) está no que o autor denomina de "aspectos de representação". Os aspectos de representação são: o resultado do processo, o processo do cliente e o processo dos empregados. O resultado do processo corresponde ao conjunto de benefícios que o cliente recebe. $\mathrm{O}$ processo do cliente está relacionado com a maneira pela qual o cliente experimenta o serviço, uma vez que ele participa do processo. Já o processo dos empregados se relaciona às operações desempenhadas pelos empregados para produzir o serviço. 


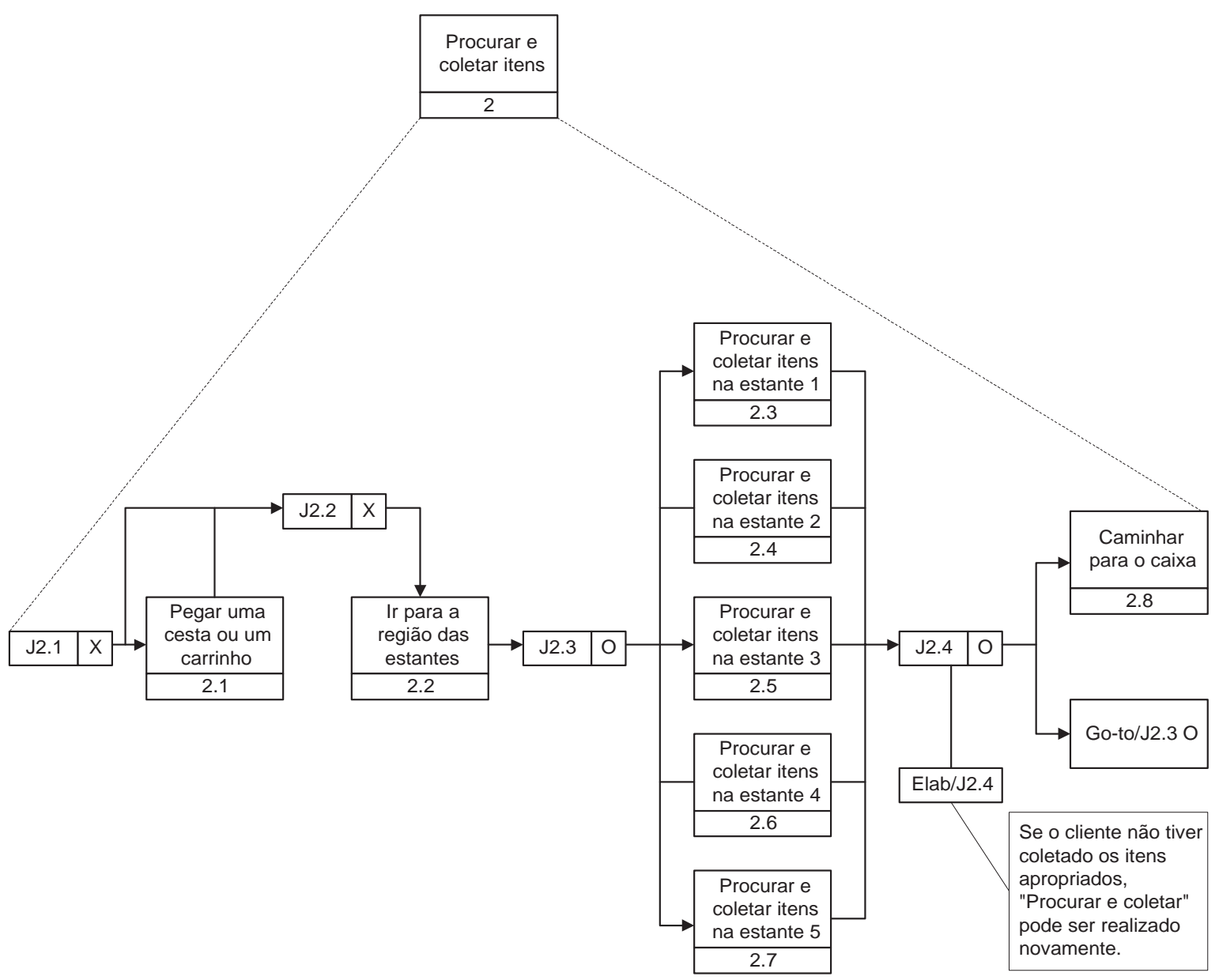

Figura 9 - Decomposição da UAC "procurar e coletar itens" Fonte: Tseng et al. (1999, p. 60)

Documento de elaboração No. Ref.:
Nome da UAC:
Rótulo da UAC:
Objeto de contato com o cliente:
Atributos do objeto de contato com o cliente:
Operações do objeto de contato com o cliente:
Relações entre o objeto e o cliente:

Figura 10 - Documento de elaboração do IDEF3 adaptado Fonte: Tseng et al. (1999, p. 57)

De acordo com Ma (1999), o projeto do serviço deve acontecer em três estágios, na seguinte ordem: desenvolvimento do serviço, desenvolvimento do processo do cliente e desenvolvimento do processo dos empregados. Depois que os benefícios para o cliente são especificados, eles são traduzidos para o projeto do processo do cliente. Em seguida, o processo dos empregados é definido com base no processo do cliente.
Cada aspecto de representação tem seu respectivo diagrama. Então, além de descrever a experiência do cliente através do diagrama de fluxo de processo do cliente, a linguagem utiliza o diagrama da definição do serviço e o diagrama de fluxo de processo dos empregados para representar os outros dois aspectos. O diagrama da definição do serviço considera os fatores estabelecidos no conceito do serviço, especificando os componentes do pacote de serviços. $\mathrm{O}$ diagrama de fluxo de processo dos empregados representa o processo do ponto de vista da empresa, o que confere à técnica a capacidade de fazer a conciliação entre as duas perspectivas (do cliente e da empresa).

O diagrama da definição do serviço faz o desdobramento do conceito do serviço nos benefícios que o cliente recebe. Este diagrama é o mais simples dentre os três diagramas, sendo de mais fácil compreensão que os outros. A Figura 11 mostra um exemplo simplificado do diagrama da definição do serviço para uma lanchonete fast-food de uma universidade. Os outros dois diagramas são bastante complexos, não sendo possível compreender nem utilizar os diagramas sem um estudo aprofundado da linguagem. 
A linguagem desenvolvida por Ma (1999) realmente representa um guia completo para projeto de serviços. Complementando as características da técnica que foram ressaltadas, ainda destaca-se o fato da linguagem incluir medidas e padrões de desempenho, o que enriquece bastante a análise do processo.

No entanto, o próprio autor afirma que os usuários devem ser sistematicamente treinados para utilizar a linguagem, devido à dificuldade de familiarização com a técnica (Ma, 1999). De fato, a representação gráfica dos diagramas de fluxo de processo do cliente e de fluxo de processo dos empregados é bastante complexa, dificultando a compreensão para usuários nãoespecialistas. Isto pode representar uma grande barreira para a utilização da técnica. Por melhor que seja, uma técnica pode não ser escolhida em relação a outra por não apresentar facilidade de uso.

\section{Comparação entre técnicas}

Ao apresentar as características de cada técnica nas seções anteriores, de certa forma se fez uma comparação entre técnicas. Porém, ainda é interessante comparar as técnicas analisadas com base nos requisitos propostos. Com este objetivo, elaborou-se a Tabela 1.

Observa-se na Tabela 1, que nenhuma técnica consegue atender completamente a todos requisitos. A linguagem de representação de Ma (1999) atende a quatro dos cinco requisitos, mas falha no requisito "facilidade de uso". O IDEF3 adaptado por Tseng et al. (1999) atende completamente a quatro requisitos e parcialmente ao requisito "suporte para a avaliação de desempenho do processo”. Considera-se que o IDEF3 adaptado por Tseng et al. (1999) atende parcialmente

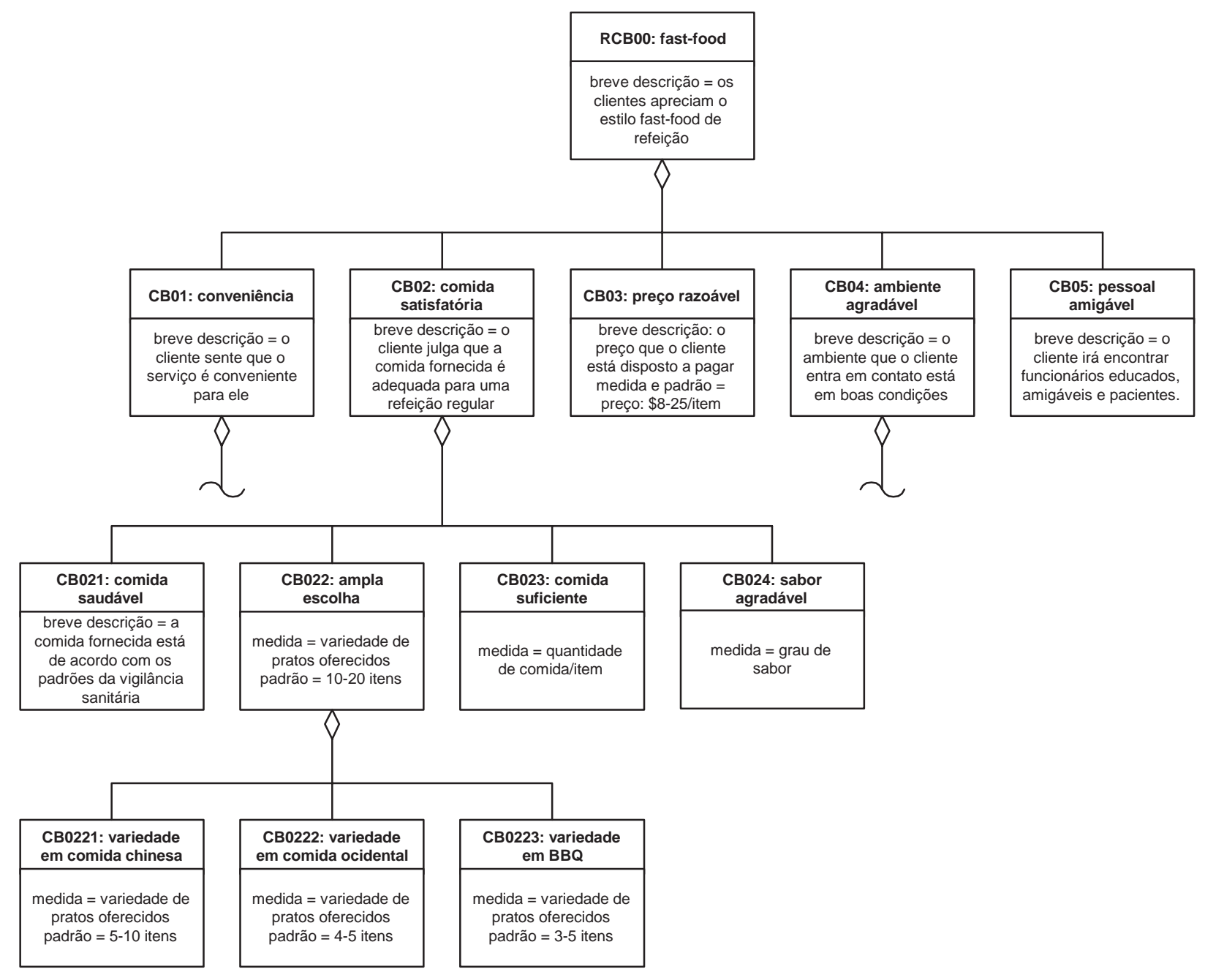

Figura 11 - Diagrama da definição do serviço para uma lanchonete fast-food Adaptado de Ma (1999) 
a este requisito porque o documento de elaboração utilizado na técnica tem a intenção de apenas descrever a atividade, embora os "atributos do objeto de contato com o cliente" contidos nele possam auxiliar a medição de desempenho.

Ao relacionar as demais técnicas com os requisitos propostos, constata-se que elas atendem a no máximo três dos cinco requisitos. A WTA e a análise da transação do serviço, mesmo dando suporte para a avaliação de desempenho, são técnicas utilizadas somente para a análise do processo. A WTA em particular, não pode ser considerada uma técnica de representação de processos, pois parte do princípio que o processo já é conhecido para que se possa fazer uma avaliação.

\section{Considerações finais}

Não se pode negar a importância da utilização de técnicas de projeto e análise de processos que sejam específicas para as operações de serviços. Mesmo assim, não é comum encontrar publicações na área que levem isto em consideração. Na prática, as técnicas de representação de processos são utilizadas somente para a análise de processos de serviços existentes, justamente porque não existe uma tradição em se projetar processos de serviços. Normalmente, os processos de serviços não são planejados, eles simplesmente "acontecem". Algumas empresas fazem um grande esforço para atingir a "excelência em serviços" utilizando ações corretivas em problemas que já ocorreram. Neste caso, o projeto do serviço é ajustado ao longo do tempo por meio de "tentativa eerro". A intenção de "fazer certo na primeira vez" não pode se tornar realidade sem que o processo do serviço seja corretamente projetado antes de ser produzido. Técnicas de representação de processos específicas para serviços podem ser úteis tanto para o projeto quanto para a análise de processos de serviços.

Ao revisar algumas técnicas de projeto e análise de processos de serviços, pode-se concluir que as mesmas foram desenvolvidas para diferentes aplicações e com diferentes objetivos. É importante considerar que todas as técnicas analisadas têm sua utilidade dentro de um contexto específico. Isto demonstra que dificilmente irá se encontrar uma técnica que se adeqüe a todas situações. Por isso, é bastante conveniente a utilização conjunta de técnicas complementares ou a adaptação de técnicas existentes. Essas alternativas procuram fechar a lacuna entre as diferentes técnicas e os requisitos desejados, conforme os objetivos do projeto e da análise do processo.

\begin{tabular}{|c|c|c|c|c|c|}
\hline \multirow[b]{2}{*}{ TÉCNICA } & \multicolumn{5}{|c|}{ REQUISITOS PROPOSTOS } \\
\hline & $\begin{array}{l}\text { 1. Adequação tanto } \\
\text { para o projeto } \\
\text { quanto para a } \\
\text { análise de } \\
\text { processos de } \\
\text { serviços }\end{array}$ & $\begin{array}{l}\text { 2. Descrição da } \\
\text { experiência de } \\
\text { serviço do ponto de } \\
\text { vista do cliente }\end{array}$ & $\begin{array}{c}\text { 3. Representação } \\
\text { gráfica baseada em } \\
\text { diagramas }\end{array}$ & $\begin{array}{l}\text { 4. Facilidade de } \\
\text { uso }\end{array}$ & $\begin{array}{l}\text { 5. Suporte para a } \\
\text { avaliação de } \\
\text { desempenho do } \\
\text { processo }\end{array}$ \\
\hline Fluxograma tradicional & 0 & $\bigcirc$ & O & O & 0 \\
\hline Service blueprint & 0 & $\mathbf{0}$ & 0 & 0 & 0 \\
\hline Mapa do serviço & 0 & $\mathbf{0}$ & 0 & 0 & $\bigcirc$ \\
\hline Estrutura de processamento de clientes & 0 & $\mathbf{0}$ & O & O & $\bigcirc$ \\
\hline IDEF0 & O & $\bigcirc$ & 0 & $\mathbf{0}$ & $\mathbf{0}$ \\
\hline Walk-through-audit & $\bigcirc$ & $\mathbf{0}$ & 0 & 0 & 0 \\
\hline Análise da transação de serviço & $\bigcirc$ & O & $\bigcirc$ & 0 & 0 \\
\hline IDEF3 adaptado & 0 & O & 0 & O & $\mathbf{0}$ \\
\hline $\begin{array}{l}\text { Linguagem de representação para } \\
\text { projeto de processos de serviços }\end{array}$ & ○ & ○ & ○ & $\bigcirc$ & ○ \\
\hline Atende ao requisito & $\mathbf{0}$ & Atende parcialmente & to requisito & ONão atend & requisito \\
\hline
\end{tabular}


16 Produto \& Produção, vol. 5, n. 3, p. 01-16, out. 2001

\section{Referências bibliográficas}

BAL, J. Process analysis tools for process improvement. The TQM Magazine. UK, v. 10, n. 5, p. 342-354, 1998.

BROWN, S. W.; FISK, R. P., BITNER; Mary J. The development and emergence of services marketing thought. International Journal of Service Industry Management. UK, v. 5, n. 1, p. 21-48, 1994.

CHASE, R. B.; STEWART, D. M. Make your service fail-safe. Sloan Management Review. USA, v. 35, n. 3, p. 35-44, spring, 1994.

CONGRAM, C.; EPELMAN, M. How to describe your service: an invitation to the structured analysis and design technique. International Journal of Service Industry Management. UK, v. 6, n. 2, p. 6-23, 1995.

FITZSIMMONS, J. A.; FITZSIMMONS, M. J. Service management: operations, strategy, and information technology. 2.ed. USA: Irwin/McGrawHill, 1998. 613p.

GIANESI, I.G. N.; CORRÊA, H. L. Administração estratégica de serviços: operações para a satisfação do cliente. São Paulo: Atlas, 1994. 233p.

HARRINGTON, H. J.; ESSELING, E. K. C.; NIMWEGEN, H. van. Business process improvement workbook: documentation, analysis, design, and management of business process improvement. USA: McGraw-Hill, 1997. 314p.

HARRINGTON, H. J. Aperfeiçoando processos empresariais. São Paulo: Makron Books, 1993. 343p.

JOHNSTON, R. Service transaction analysis: assessing and improving the customer's experience. Managing Service Quality. UK, v. 9, n. 2, p. 102109, 1999.

KETTINGER, W.; TENG, J. T. C., GUHA, S. Business process change: a study of methodologies, techniques, and tools. MIS Quarterly. USA, v.21, n. 1, p. 55-80, march, 1997.

KINGMAN-BRUNDAGE, J.; GEORGE, W. R.; BOWEN, D. E. "Service logic": achieving service system integration. International Journal of Service Industry Management. UK, v. 6, n. 4, p. 20-39, 1995.

KOLJONEN, E. L. L.; REID, R. A. Walk-through audit provides focus for service improvements for Hong Kong law firm. Managing Service Quality. UK, v. 10, n. 1, p. $32-45,2000$.
LOVELOCK, C. H. Product plus: produto + serviço = vantagem competitiva. São Paulo: Makron Books, 1995. 476p.

MA, Q. An approach to the representation of service process design. Hong Kong, 1999. 203p. Thesis (Doctor of Philosophy in Industrial Engineering and Engineering Management) - Department of Industrial Engineering and Engineering Management, Hong Kong University of Science and Technology.

MAYER, R. J., et al. A framework and a suite of methods for business process reengineering. Disponível na internet. http://www.idef.com/articles/ framework/. 21 julho 1999.

MIERS, D. Uso de ferramentas e tecnologia nos projetos de RPE. In: COULSON-THOMAS, Colin J. (org.) Reengenharia dos processos empresariais: mito e realidade. Rio de Janeiro: Record, 1996. 293p.

PLAIA, A.; CARRIE, A. Application and assessment of IDEF3 - process flow description capture method. International Journal of Operations \& Production Management. UK, v. 15, n. 1, p. 63-73, 1995.

RAMASWAMY, R. Design and management of service processes: keeping customers for life. USA: Addison-Wesley, 1996. 424p.

ROTONDARO, R. G.; OLIVEIRA, C. L. de. O indicador do potencial de falha como ferramenta de melhoria da qualidade do serviço. In: ENCONTRO NACIONAL DE ENGENHARIA DE PRODUÇÃO, 19., Rio de Janeiro - RJ, 1999. Anais... Rio de Janeiro: UFRJ, 1999. CD-ROM.

SCHMENNER, R. W. Service operations management. USA: Prentice Hall, 1995. 406p.

SHOSTACK, G. Lynn. Designing services that deliver. Harvard Business Review. USA, v. 62, n. 1, p. 133139, january-february, 1984.

Service positioning through structural change. Journal of Marketing. USA, v. 51, n. 1, p. 34-43, january, 1987.

SLACK, N. et al. Administração da produção. São Paulo: Atlas, 1997. 726p.

TÉBOUL, J. A era dos serviços: uma nova abordagem de gerenciamento. Rio de Janeiro: Qualitymark Ed., 1999. 295p.

TSENG, M. M.; QINHAI, M.; SU, C. Mapping customers' service experience for operations improvement. Business Process Management Journal. UK, v. 5, n. 1, p. 50-64, 1999. 